\title{
Infectious epithelial keratitis
}

INSERM

\section{Source}

INSERM. (1999). Orphanet: an online rare disease and orphan drug data base. Infectious epithelial keratitis. ORPHA:137593

Infectious epithelial keratitis is a rare, potentially sight-threatening, acquired ocular disease chracterized by corneal epithelium inflammation resulting from viral (mainly Herpes Simplex virus), bacterial, fungic or protist infection, manifesting with variable symptoms, such as conjunctival hyperemia, lacrimation, rapid onset of pain, blurred vision and/or photophobia, depending on the causative agent. 